\section{Gently moving teeth into the ideal position}

GEO is a European orthodontic laboratory with 20 years of experience in continuous research and innovation in exclusive modern orthodontics.

In 2010, GEO expanded its range of orthodontic appliances by introducing NUVOLA, a transparent aligner system that facilitates gradual and painless teeth straightening to address functional, aesthetic and other orthodontic issues. Each aligner is designed to move the teeth with gentle forces into the ideal position.

All dealings with the lab are via NuvolaWEB, an easy to use online case management interface. Once registered, clinicians can manage all their cases online and get a rapid response to any queries from the lab as well as monitor the progress of cases through each stage of the production process to final delivery to the surgery.

The aligners are delivered within ten working days of confirmation of the treatment plan and delivery to your surgery is by courier at no cost to you.

GEO offer full training support and their next NUVOLA training course is in London on 10 June. It costs just $£ 125$ and a $£ 100$ rebate is offered to delegates off their first case with NUVOLA.

To find out more contact info@geoorthodontic.com or visit www. geoorthodontic.com.
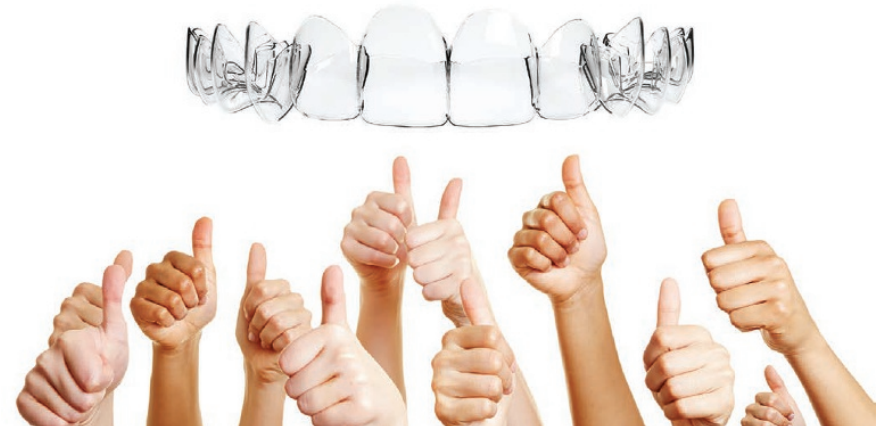

\section{Numerous products for prophylaxis and restoration}

ContactPro is the new matrix system from Microbrush for Class II restorations. It includes bands, rings, wedges and high-quality stainless steel precision instruments. One special feature is the ring with 'memory effect' - it retains both a constant clamping force and a perfect fit even after numerous applications, thus minimising the formation of excess composite. The SmartView matrix clip guarantees unrestricted visibility and thus facilitates access to the work area. There are three different kits available containing the necessary equipment.

Young Dental is the successful line of professional teeth-cleaning products. The portfolio includes polishing cups, disposable prophy angles and pastes for polishing and removing surface discoloration. The exceptional efficiency, superior brightening results and neutral $\mathrm{pH}$ that these offer speak for themselves.

The popular Zooby brand is at the forefront when it comes to professional cleaning of children's teeth. The colourful prophylaxis products are decorated with animals ranging from A for alligator through to $\mathrm{Z}$ for zebra. This ensures a relaxed atmosphere, facilitates anxiety-free treatment and creates a positive foundation for the child's next visit to the dentist. Young patients can choose from a wide variety of polishing pastes in popular flavours.

\section{Intelligent obturation}

With ROEKO GuttaFlow bioseal, Coltene is providing endodontists with an intelligent obturation material that does more than simply seal and fill the root canal.

Upon contact with fluids, the bioactive material provides the tooth with natural repair constituents, such as calcium and silicates - improving canal regeneration and treatment success.

The idea is remarkably simple: after curing, the new GuttaFlow bioseal forms hydroxyapatite crystals on the surface which significantly improve adhesion and actively encourage the regeneration of bone and dentine tissue. This helps practitioners provide long-lasting solutions for their patients.

In the past, the only materials that exhibited the same curative properties of GuttaFlow bioseal were MTA or bioglass - but these materials involved a long curing time and complicated handling. These difficulties are eliminated with Coltene's innovative bioseal, which is both easy to use and fast curing (12-16 minutes).

Whether practitioners are experienced in endodontic treatments or just starting out, ROEKO GuttaFlow bioseal from Coltene provides predictable and effective results. To find out more, contact the expert Coltene

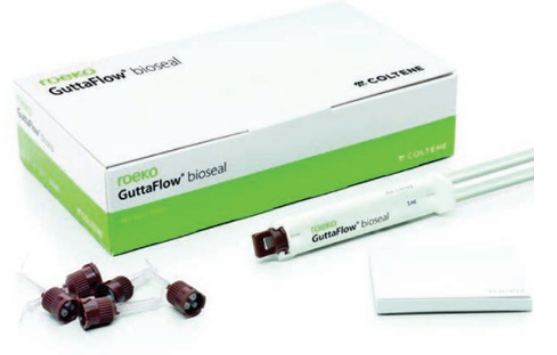
team today.

To find out more about Coltene products, visit www.coltene.com.
Another innovation is Reflective DryTips from Microbrush. These parotid pads aid in controlling moisture during treatment. They are the ideal supplement to saliva ejectors and cotton rolls, because the extremely absorbent pulp pads are placed on the inside of the cheek, right in front

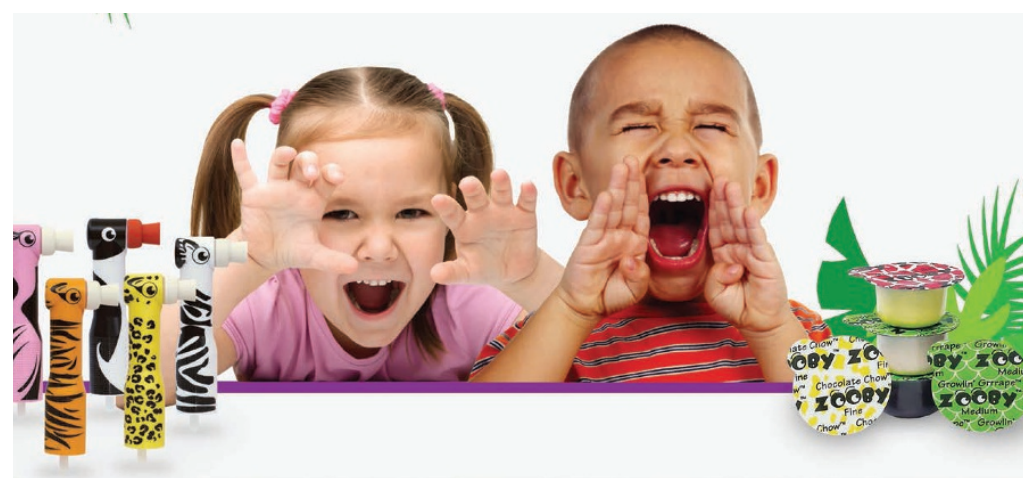

of the largest salivary gland, and therefore keep the work area dry. The pads are coated with a silver film on one side, which is aligned with the dentition. This generates significantly more brightness and better visibility, which is advantageous when working with a microscope, for example.

More info on all these products via Young Innovations: www.ydnt.com 